\title{
Mycoplasma origin of tumor cell protein
}

To the editor-In the November 1997 issue, Matsumoto et al. described a novel human protein (M161 Ag) expressed by some tumor cell lines but not found in normal human tissues, and suggested a potentially important mechanism for destruction of tumor cells by autologous complement ${ }^{1}$. We wish to point out the pronounced similarities between M161Ag and a mycoplasma protein originally described because of its effects on cultured cells ${ }^{2}$.

We searched for similarities between the two quoted cDNA and predicted amino acid sequences. Not only is the $5^{\prime}$ PCR primer identical to the previously reported Mycoplasma fermentans $\mathrm{p} 48$ sequence ${ }^{2}$, but the first $570 \mathrm{bp}$ of the $\mathrm{M} 161 \mathrm{Ag}$ sequence is identical to the previously described sequence, with the exception of three single base changes and a single base insertion. As would be expected, the encoded proteins also show extensive similarity-of the first 114 amino acids of $\mathrm{M} 161 \mathrm{Ag}, 112$ are identical to that of $M$. fermentans and the subsequent amino acid sequence up to residue 186 of M161Ag can also be explained by addition of a single $\mathrm{T}$ nucleotide at base pair 404 of the mycoplasma sequence.

Although in a note added in proof Matsumoto et al. refer to some homology between $\mathrm{M} 161 \mathrm{Ag}$ and a mycoplasma sequence, the above described similarity is such that their $M 161 \mathrm{Ag} \mathrm{cDNA}$ sequence is almost certainly of mycoplasma origin. As previously described ${ }^{2}$ this could be tested by Southern blot analysis with a M161Ag probe, hybridised onto genomic DNA prepared from normal human tissue, rather than cell lines which may contain mycoplasma contamination. The paper by Matsumoto et al. may be telling us more about mycoplasma biology than human tumor immunology!

\section{B.F. FlanAGAN, D.J. NEWTON \\ \& P.M. JOHNSON \\ Department of Immunology \\ Duncan Building \\ University of Liverpool Medical School \\ Daulby Street \\ Liverpool L69 3GA, UK}

Matsumoto et al. reply-We focused on the novelty of the protein functions in malignancy as present in leukemia patients. Although at the time of submission we failed to detect any homologies to released GenBank sequences, we did present a note added in proof revealing partial homology to a M.fermentans product called p48 (ref. 2). As an obvious next step we further investigated homology to our new protein M161Ag and discovered that the gene is exogenously transferred to M161Ag-positive cell lines and that $\mathrm{M} 161 \mathrm{Ag}$ is indeed a mycoplasma product. A more complete account of this work and the structural and functional properties of M161Ag has been submitted for publication.

Indeed the genes $M 161 \mathrm{Ag}$ and $p 48$ are highly homologans but the corresponding proteins are different. M161Ag remains the only identified mycoplasma protein involved in complement activation. Mycoplasma products have recently been demonstrated to have anti-tumor and growth inhibitory properties ${ }^{3,4}$ and we and others have detected M161Ag and M.fermentans in leukemia and AIDS $^{5}$ patients respectively. Irrespective of its origin, our report suggests that under certain circum- stances, if malignant cells start expressing M1651Ag, the cells will be discrimminated as non-self via homologans complement as in HIV-infected cells. Our study also draws attention to the impact of parasitic proteins on host immune modulation.

\section{T. Seya , N.A. Begum \& M. Matsumoto Department of Immunology \\ Osaka Medical Center for Cancer and \\ Cardiovascular Diseases \\ Higashinari-ku, Osaka 537 Japan}

1. Matsumoto, M. et ol. A novel protein that participates in non-self discrimination of malignant cells by homologous complement. Nature Med. 3, 1266-1270 (1997).

2. Hall, R.E. et al. CDNA and genomic expression of the P48 monocytic differentiation/ activation factor, a Mycoplasma fermentans gene product. Biochem. I. 319, 919-927 (1996).

3. Taylor-Robinson, D. et al. Mycoplasmas and oncogenesis. Lancet 347, 1555-15556 (1996).

4. Tsai, S. et al. Mycoplasmas and oncogenesis: persis tent infection and multistage malignant transformation. Proc.Natl.Acad.Sci. U.S.A. 92 10197-10201 (1995).

5. Lo, S.C. et al. Enhancement of HIV-1 cytocidal effects in CD4+ lymphocytes by the AIDS-associated mycoplasma. Science 251, 1074-1076(1991).

\section{Therapeutic replication-competent herpes virus}

To the Editor-In a December News \& Views article, Martuza discussed the potential of selectively replication proficient herpes simplex virus as a cytotoxic agent in the treatment of malignant brain tumors' ${ }^{1}$. We report the first clinical trials.

We have characterized the HSV mutant 1716 which is only capable of replication in proliferative cells ${ }^{2}$. HSV1716 is safe when inoculated intracerebrally in rodents and has demonstrated wide ranging anti-tumor activity in xenograft models ${ }^{3}$.

We have initiated an MRC-funded, approved, phase I, dose escalation study of HSV 1716 in patients with recurrent glioblastoma who have failed all conventional treatments. The study is designed along conventional lines with successive cohorts of three patients receiving increasing doses of virus by direct intra-tumor injection. Patients have been treated at a starting dose of $10^{3}$ pfu with no adverse sequelae noted after the first few weeks.

Since 1716 is replication competent it has the theoretical potential to cause encephalitis, although no evidence of encephalitis was seen in rodent studies. HSV 1716 is Acyclovir/Gancyclovir sensitive but to date, administration has not been required since no evidence of encephalitis has been seen in any patient.

We believe that animal experiments give little information with respect to the ultimate safety, toxicity or efficacy of potentially therapeutic viruses in humans. This is especially true for a neurotropic, replication competent virus whose clinical course will be modified at least by the immune system. We therefore believe that progression to human trials of 1716 is totally justified in patients whose disease offers no prospect of even modest survival and who are fully informed of the experimental nature of the procedure.

1. Martuza, R.L. Act locally, think globally. Nature Med. 12, 1323 (1997).

2. Maclean, A.R. et al. Herpes simplex virus type 1 deletion variants 1714 and 1716 pinpoint neurovirulence related sequences in Glasgow strain $17+$ between immediate early gene 1 and the ' $a$ ' sequence. J. Gen. Virol. 72, 631-639 (1991).

3. Kesari, S. et al. A mutant herpes simplex virus replicates in brain tumours but not in neurons derived from a human embryonal carcinoma cell line. Lab. Invest. 73, 636-648 (1995).

R. Rampling ${ }^{1}$, G. Cruickshank ${ }^{2,3}$, A. MacLean ${ }^{4} \&$ M. Brown ${ }^{5}$

${ }^{1}$ Glasgow University Dept. of Clinical Oncology, Beatson Oncology Centre, Glasgow G116NT, UK

${ }^{2}$ Glasgow University Dept. of Neurosurgery, Southern General Hospital, Glasgow G514TF, UK

${ }^{3}$ Current address: University Dept. Neurosurgery, Queen Elizabeth Hospital, Birmingham, UK

"Glasgow University Division of virology, Institute of Virology, Glasgow G11 5JR, UK

${ }^{5}$ Neurovirology Research Laboratories, Institute of Neurological Science, Glasgow G51 4TF, UK 